\title{
Graph clustering in industrial networks
}

\author{
V. Bouet • A.Y.Klimenko
}

March 2019

\begin{abstract}
The present work investigates clustering of a graph-based representation of industrial connections derived from international trade data by Hidalgo et al (2007) and confirms existence of around ten industrial clusters that are reasonably consistent with expected historical patterns of diffusion of innovation and technology. This supports the notion that technological development occurs in sequential innovation waves. The clustering method developed in this work follows conceptual ideas of Lambiotte and Barahona (2009), who suggested to use random walk to assess a hierarchical structure of network communities where different levels of the hierarchy correspond to different diffusion times. We, however, implement these ideas differently to match physics of the problem under consideration and introduce a hierarchal clustering procedure that is combined with convenient resorting of the elements. An equivalent spectral interpretation of the clustering is also given and discussed in the paper. Graph clustering, random walk, diffusion of innovation and technology
\end{abstract}

\section{Introduction}

The classical view of economics, which can be traced back to Adam Smith and David Ricardo, is firmly based on equilibrating economic forces presuming that, when disturbed, economic equilibrium is promptly restored by these forces (Freeman \& Soete, 1997). There is, however, an alternative view introduced by Schumpeter (1947), who explained the existence of Kondratiev cycles in economic activity by a sequence of technological revolutions. These revolutions are instigated by a surge of innovations that move economy away from equilibrium by eliminating obsolete technologies and thus creating conditions favorable for rapid economic progress (i.e. Schumpeter's concept of "creative destruction"). After a technological surge, higher profit margins tend to persist in innovative sectors for a substantial time until these sectors reach maturity and the wave fades away.

While, according to the classical interpretation, economy drives technological development, the alternative view is that technological progress plays the leading role over longer periods of time and create technological waves modulating economic growth. The inquisitive reader may note that technological waves of appreciable magnitude are possible only if different innovations are somehow connected to each other a single innovation would rapidly blend into the economy and cannot create a lasting disturbance of the equilibrium. Existence of industrial clusters is, thus, a principal question that may pose a strong argument in favour of the Schumpeterian interpretations. Hidalgo et al. (2007) and Hausmann et al. (2014) have recently introduced a theory and a method that allows us to examine links and connections between different industries by analysing global export data. This theory relates industrial connections to international trade data and demonstrates that industrial development tends to take place within connected proximities of existing industries (Hidalgo et al. 2007). This process can be interpreted as diffusion of resources and innovation between connected sectors of industry, which can be modelled by a random walk on graphs representing industrial connections. The fact that these data are readily available from the United Nations databases makes a strong argument in favour of this method. The links between industries seem to indicate the presence of industrial clusters (see the graph shown in the next chapter). The goal of the present work is

SoMME, The university of Queensland, Qld 4072, Australia, email: klimenko@mech.uq.edu.au 
a more rigorous and formal analysis of the existence and structure of clusters for the undirected industrial network introduced by Hidalgo et al. (2007) and Hausmann et al. (2014).

A large number of publications is dedicated to graph partitioning and identification of communities (Fortunato, 2009, Porter et al., 2009, Mucha et al., 2010, Jeub et al., 2015, Fortunato \& Hric, 2016, Lyzinski et al., 2017). The spectral method, based on using the Fiedler vector, seems to be the most popular but, as Fortunato \& Hric (2016) remark, the problem of identifying network communities is ill-defined — there is no universal definition or algorithm that determines communities in some unique or undisputedly superior way. The method of identifying communities needs to reflect a physical understanding of the problem. In the present case, this implies the involvement of random walks, since diffusion on the network is expected to reflect the process of diffusion of innovation within and between the industrial clusters (Hidalgo et al. 2007). The literature dedicated to random walks is vast; Aldous \& Fill (2014) and Masuda et al. (2017) presented the excellent reviews of random walks on graphs, while Grimmett (2018) overviews a broader spectrum of issues associated with probabilistic processes in networks. In general, it would be productive to consider time-dependent or multisliced networks (Mucha et al., 2010, Holme \& Saramaki, 2012, Bassett et al., 2013; Holme, 2015, Masuda \& Lambiotte, 2016), since links between industries obviously evolve in time. However, detailed historical information about the evolution of these links does not exist and, therefore, all links are necessarily treated as time-independent.

In many respects, the present analysis is similar to the concept of partition stability introduced in Lambiotte \& Barahona (2009) and extended in more recent publications Delvenne et al. (2010); Schaub et al. (2012); Lambiotte et al. (2015) to involve both discrete and continuous time as well as other generalisations (e.g. Rosvall et al., 2014, Salnikov et al., 2016). As suggested by Lambiotte et al. (2015), we use discrete-time random walks (i.e. a Markov chain) to analyse the community structure of a network, which corresponds to different resolution levels for different diffusion times. This approach allows us to examine hierarchies of communities, which have been discussed in a few publications that, generally, may or may not be related to random walks (e.g. Lancichinetti et al., 2009, Mucha et al., 2010, Jeub et al., 2015, Lambiotte et al., 2015. Lyzinski et al., 2017). There are, however, some essential differences between our work and the partition stability approach. First, we understand and define clusters differently from Lambiotte \& Barahona (2009) - this is determined by our physical interpretation of the industrial networks introduced by Hidalgo et al. (2007). This difference is explained further in Section 3. Second, the stability of a partition is suggested by Lambiotte \& Barahona (2009) as a criterion, which is designed to assess the quality of a community partition produced by other algorithms, while we use our approach as both a definition of clusters and an algorithm producing a hierarchy of partitions and a convenient ordering of the nodes.

Our approach has both useful transitional and conceptually transparent spectral interpretations. It allows us to identify nine primary industrial clusters, which are reported in the last sections of this work. Although designed for a specific problem, the clustering procedure introduced in the present work is generic and, at least in principle, can be used in different applications (and, possibly, with different clustering criteria). This procedure is not intended to enforce the fastest possible division of a graph into a given number of clusters, but to follow the mechanics of diffusion of innovation explained above, and examine the complex structure of connections created by this diffusion.

\section{Proximity of industries and international trade.}

The theory of proximity of different industrial products, introduced by Hidalgo et al. (2007), statistically reflects correlations between success of a country in trading a certain product and success in trading other products that are technologically associated with the first product. If $X_{\alpha j}$ represents export of product $j$ by country $\alpha$ then the quantity

$$
\tilde{X}_{\alpha j}=\frac{1}{\tilde{Y}_{\alpha}} \frac{X_{\alpha j}}{\sum_{\beta} X_{\beta j}}, \quad \tilde{Y}_{\alpha}=\frac{Y_{\alpha}}{\sum_{\beta} Y_{\beta}}, \quad Y_{\alpha}=\sum_{j} X_{\alpha j}
$$

which is called revealed competitive advantage, represents the fraction that a particular country $\alpha$ contributes to the world trade in exporting a particular product $j$ related to $\tilde{Y}_{\alpha}$ - the fraction of all exports of this country in the world trade. Values of $\tilde{X}_{\alpha j} \geq 1$ indicate that country $\alpha$ has a comparative advantage in exporting product $j$. The index-function

$$
M_{\alpha j}= \begin{cases}1, & \tilde{X}_{\alpha j} \geq 1 \\ 0, & \tilde{X}_{\alpha j}<1\end{cases}
$$


indicates whether country $\alpha$ is competitive in exporting product $j$. Proximity, which is defined by

$$
\varphi_{j i}=\sum_{\alpha} \frac{M_{\alpha j} M_{\alpha i}}{\max \left(K_{j}, K_{i}\right)}, \quad K_{j}=\sum_{\alpha} M_{\alpha j}
$$

is similar to the matrix of correlation coefficients between columns of the matrix $M_{\alpha j}$. Proximity defined by (3) is symmetric and constrained $0 \leq \varphi_{j i} \leq 1$ where $\varphi_{j i}=1$ only if the columns $j$ and $i$ are the same. Large values of the proximity $\varphi_{j i} \sim 1$ indicate that a country producing product $j$ also tends to produce product $i$ and, hence, products $i$ and $j$ are very likely to be related. Small values of $\varphi_{j i}$ do not tell us anything specific since small variations of $\varphi_{j i}$ are likely to be coincidental, not revealing anything about real-world links between the products. We wish to consider only connections between products that have largest proximities and ignore proximities of smaller magnitudes - only large values of $\varphi_{j i}$ are indicative of a technological or logistic connection between the products. This, however, would result in a disconnected graph. Therefore, we follow Hidalgo et al. (2007) and use a two-stage procedure. First, Kruskal's algorithm is used to connect $N$ product nodes by a tree with $N_{1}=N-1$ edges. This algorithm selects largest proximities one by one and connects the corresponding nodes only if this connection does not form a loop. In the second stage, the $N_{2}$ largest values $\varphi_{j i}$, that were not used as connectors in the first stage, are selected to form additional connections. The result is a complex undirected graph, which is shown in Figure 1 . This figure seems to indicate presence of 6 clusters outlined by red rectangles. The present work investigates the existence of clusters in this graph by introducing and using a more rigorous procedure for cluster analysis.

\section{Defining clusters by using a random walk}

Our definition of clusters is necessarily based on random walks on the graph specified in the previous section since it is this random walk that, according to Hidalgo et al. (2007), emulates the rate of diffusion of technological innovations between industrial sectors. Mathematically, random walk is represented by a discrete Markov chain (see Grinstead \& Snell, 1997)), which is characterised by single-step transitional probabilities $P_{j i}$. The distribution of probabilities at time step $t$ denoted by $p_{i}(t)$ satisfies

$$
p_{j}(t+1)=\sum_{i} P_{j i} p_{i}(t) \text { or } \mathbf{p}(t+1)=\mathbf{P} \mathbf{p}(t)
$$

if the vector - matrix notation is used. The transitional probabilities are specified by

$$
\mathbf{P}=(1-\beta) \mathbf{T}+\beta \mathbf{I}, \quad \mathbf{T}=\mathbf{A D}^{-1}
$$

where $\mathbf{A}$ is an $N \times N$ symmetric adjacency matrix: $A_{j i}=1$ if nodes $i$ and $j$ connected and $A_{j i}=0$ otherwise $\left(A_{i i}=0\right), T$ is its version subject to the probability-preserving normalisation, $\mathbf{D}$ is a diagonal matrix with $D_{i i}=d_{i}$ specifying the degree of node $i, \mathbf{I}$ is the unity matrix, and $N$ is the number of nodes in the graph. The parameter $\beta$ specifies the probability of a randomly walking particle to remain at the same node. As discussed in the next section, $\beta=1 / 2$ is a suitable, safe choice for this parameter. The normalisation of the transition matrix preserves the overall probability $\Sigma_{i} p_{i}=1$. It is possible to consider random-walk transition probabilities proportional to the proximities $\varphi_{j k}$ of the connected nodes but this would not substantially affect the results and we use the simpler definition of the adjacency matrix given above. The matrix $P_{j i}^{n}$ defined by

$$
\mathbf{P}^{n}=\underbrace{\mathbf{P} \mathbf{P} \ldots \mathbf{P}}_{n \text { times }}
$$

specifies the probability distributions $\left(p_{j}\right)_{i}^{n}=P_{j i}^{n}$ evolved from the initial localisation at node $i$ after $n$ time steps.

The definitions of partition stability given by Lambiotte et al. (2015) interpret clusters as subgraphs that tend to retain a random walk for a given number of time steps. This definition involves maximisation of stability - a certain quantity defined on the basis of the $n$-step transitional probabilities $P_{j i}^{n}$ and stationary distributions $p_{j}^{\circ} \equiv d_{j} /(2 E)$, where $E$ is the number of edges. In simple terms, a partition into clusters after $n$ time steps is considered to be of good quality when, on average, $P_{j i}^{n}$ is relatively large (above the stationary distribution when properly scaled) if $i$ and $j$ belong to the same cluster, and is relatively small if $i$ and $j$ are not from the same cluster. That implies that clusters are expected to have minimal interactions. Our understanding of clusters does not require that members of an industrial cluster must not significantly 
interact with other industries (which would not seem justified) but that, after $n$ steps, these interactions become similar for all members of the same cluster. Therefore, after formation of a cluster, the cluster members are expected to display similar evolutionary dynamics.

Our interpretation of clustering can be expressed in terms of the similarity relation given in the following proposition

Proposition 1 Two nodes $i$ and $j$ are deemed to be similar $\mathbf{p}_{i}^{n} \cong \mathbf{p}_{j}^{n}$ and belong to the same cluster at the time step $n$ provided the probability distributions originated from these nodes (i.e. $\mathbf{p}_{i}^{n}$ and $\mathbf{p}_{j}^{n}$ ) are similar for this and the subsequent time steps.

The similarity, which is denoted by $\mathbf{p}_{i}^{n} \cong \mathbf{p}_{j}^{n}$ here and in the rest of the paper, can be defined in different ways. The definition

$$
R_{i j}^{n}=\mathbf{q}_{i}^{n} \cdot \mathbf{q}_{j}^{n}=\left(\mathbf{q}_{i}^{n}\right)^{\mathrm{T}}\left(\mathbf{q}_{j}^{n}\right) \geq 1-\delta, \quad\left(q_{k}\right)_{i}^{n} \equiv \sqrt{\left(p_{k}\right)_{i}^{n}}
$$

with a sufficiently small threshold $\delta$ is used due to its practical stability and simplicity of avoiding the need to renormalise vectors since $\mathbf{q}_{i}^{n} \cdot \mathbf{q}_{i}^{n}=1$ for any $i$ because of the probability normalisation. Conceptually, this or any other choice evaluating a quantity similar to a correlation coefficient between $\mathbf{p}_{i}^{n}$ and $\mathbf{p}_{j}^{n}$ would be suitable to define similarity $\mathbf{p}_{i}^{n} \cong \mathbf{p}_{j}^{n}$.

Note that the initial conditions at $t=0$ correspond to $\mathbf{P}^{0}=\mathbf{I}$ and, consequently, $R_{i j}^{0}=0$. Assuming that the graph under consideration is connected and $\mathbf{P}$ is irreducible, the opposite limit of $t=n \rightarrow \infty$ implies that $\mathbf{P}^{n} \rightarrow \mathbf{P}^{\infty}=\left[\mathbf{p}^{\circ}, \ldots, \mathbf{p}^{\circ}\right]$. That is distributions become stationary irrespective of the initial localisation: $\mathbf{p}_{i}^{n} \rightarrow \mathbf{p}^{\circ}$ or, with the use of the component notations, $P_{j i}^{n} \rightarrow p_{j}^{\circ} \equiv d_{j} /(2 E)$ for any $i$ and $n \rightarrow \infty$. Note that $R_{i j}^{n} \rightarrow 1$ as $t=n \rightarrow \infty$ since all columns of $\mathbf{P}^{n}$ become fully correlated. In this work, we consider only connected graphs since, otherwise, clustering can be performed independently for each of the connected components. The graphs under consideration are undirected (and thus satisfy the detailed balance $\left.P_{j i} p_{i}^{\circ}=P_{i j} p_{j}^{\circ}\right)$ but, in practice, the procedure given here may tolerate some degree of directionality. The details of this procedure are considered further in this section .

The original ordering of the nodes may not be the best to represent cluster hierarchies. The clustering procedure is accompanied by reordering of the elements. For the purposes of this procedure, single nodes are considered to be clusters containing a single element. Clusters, say $\mathfrak{A}$ and $\mathfrak{B}$ that have the corresponding numbers of elements (nodes) $N_{\mathfrak{A}}$ and $N_{\mathfrak{B}}$, are considered to be similar $\mathfrak{A} \cong \mathfrak{B}$ provided

$$
\bar{R}_{\mathfrak{A} \mathfrak{B}}^{n} \equiv \frac{1}{N_{\mathfrak{A}} N_{\mathfrak{B}}} \sum_{i \in \mathfrak{A}} \sum_{j \in \mathfrak{B}} R_{i j}^{n} \geq 1-\delta
$$

where $R_{i j}^{n}$ is defined by (7). Jointly with (8), this definition somewhat resembles the Frobenius inner matrix product. At $N_{\mathfrak{A}}=N_{\mathfrak{B}}=1$, definition (8) is obviously consistent with the previously defined similarity of the nodes. The limiting value $\bar{R}_{\mathfrak{A} \mathfrak{B}}^{n}=1$ is achieved if and only if the clusters $\mathfrak{A}$ and $\mathfrak{B}$ are composed of identical elements $\mathbf{p}_{i}=\mathbf{p}_{j}$ for all $i, j \in \mathfrak{A} \cup \mathfrak{B}$. Note that merging preserves self-similarity of the clusters, that is, if $\bar{R}_{\mathfrak{A} \mathfrak{B}}^{n} \geq 1-\delta, \bar{R}_{\mathfrak{A} \mathfrak{A}}^{n} \geq 1-\delta$ and $\bar{R}_{\mathfrak{B} \mathfrak{B}}^{n} \geq 1-\delta$, then $\bar{R}_{\mathfrak{C} \mathfrak{C}}^{n} \geq 1-\delta$ where $\mathfrak{C}=\mathfrak{A} \cup \mathfrak{B}, \mathfrak{A} \cap \mathfrak{B}=\varnothing$ and $N_{\mathfrak{C}}=N_{\mathfrak{A}}+N_{\mathfrak{B}}$. Indeed,

$$
\begin{aligned}
1 & \geq \bar{R}_{\mathfrak{C} \mathfrak{C}}^{n}=\frac{N_{\mathfrak{A} \mathfrak{A}}^{2} \bar{R}_{\mathfrak{A} \mathfrak{A}}^{n}+2 N_{\mathfrak{A}} N_{\mathfrak{B}} \bar{R}_{\mathfrak{A} \mathfrak{B}}^{n}+N_{\mathfrak{B} \mathfrak{B}}^{2} \bar{R}_{\mathfrak{B} \mathfrak{B}}^{n}}{N_{\mathfrak{C}}^{2}} \\
& \geq \underbrace{\frac{N_{\mathfrak{A} \mathfrak{A}}^{2}+2 N_{\mathfrak{A}} N_{\mathfrak{B}}+N_{\mathfrak{B} \mathfrak{B}}^{2}}{N_{\mathfrak{C}}^{2}}}_{=1} \underbrace{\min \left(\bar{R}_{\mathfrak{A} \mathfrak{A}}^{n}, \bar{R}_{\mathfrak{A} \mathfrak{B}}^{n}, \bar{R}_{\mathfrak{B} \mathfrak{B}}^{n}\right)}_{\geq 1-\delta} \geq 1-\delta
\end{aligned}
$$

The clusters that are similar are merged with preservation of the original ordering within and between the merged clusters. The overall ordering of elements, which is represented by ordering of the clusters and ordering of elements within the clusters, is nevertheless changed as the clusters merge: subsequent similar clusters are moved to their first similar cluster to achieve a merger. Note that the similarity of the clusters is, generally, not transitive, that is

$$
\mathfrak{A} \cong \mathfrak{B} \cong \mathfrak{C} \not \mathfrak{A}
$$

is a possibility for some set of clusters $\mathfrak{A}, \mathfrak{B}$ and $\mathfrak{C}$. That is, $\mathfrak{A}$ and $\mathfrak{C}$ are both similar to $\mathfrak{B}$ and must be reasonably similar to each other but may or may not be similar according to formal definition (8). If $\mathfrak{C} \nsucceq \mathfrak{A}$, the outcome of merging of $\mathfrak{A}, \mathfrak{B}$ and $\mathfrak{C}$ is dependent on the initial ordering (i.e. whether $\mathfrak{A}$ and $\mathfrak{B}$ or $\mathfrak{B}$ and 
$\mathfrak{C}$ are merged first). However, the algorithm we use forms clusters independently of the initial ordering of the nodes. This can be achieved: 1) by transitive closure of the similarity relation (i.e. enforcing $\mathfrak{C} \cong \mathfrak{A}$ in example (9) or 2) by ordering similarities between clusters, performing merges of more similar clusters first and then re-evaluating similarity. Transitive closure is more simple and, by default, is used in the present work. Hence, the practical algorithm used here allows for slight compromises over condition (8) and this does not seem to cause any practical problems (multiple mergers are infrequent and violations of (8) are small).

The algorithm considered here does not produce a unique ordering of the nodes - the final ordering remains dependent on the initial ordering. Clusters are formed independently of ordering of the elements and different orderings produced by the algorithm are suitable to visualise clusters (best appearance is a subjective matter). If the requirement of producing a unique ordering at the end is imposed, the nodes need to be pre-ordered by another fixed algorithm - say by using ordering of the conventional Fiedler vector - and then further ordered by the clustering algorithm. The Fiedler vector corresponds to second smallest eigenvalue (non-zero in this case) of the Laplacian matrix $\mathbf{L}=\mathbf{D}-\mathbf{A}$ (Fortunato, 2009). Note that the Fiedler vector outlines the least connected (and therefore the slowest converging) component of the graph while the clustering algorithm considers a hierarchy of clusters that correspond to different characteristic times.

The procedure specified in this section has not been optimised for speed and various measures can be implemented to speed up the simulations. For example, replacing $\mathbf{P}^{n+1}=\mathbf{P} \mathbf{P}^{n}$ by $\mathbf{P}^{2 n}=\mathbf{P}^{n} \mathbf{P}^{n}$ would double the time step or, generally, $R_{i j}^{n}$ does not need to be evaluated for all $i$ and $j$. The speed, however, was not a pertaining issue in the present simulations.

The physical interpretation of clustering is transparent - two nodes belong to the same cluster if they are well-connected and the distinction between random walks originated at these nodes disappears at a given time step. This definition, obviously, depends on time: as clusters grow and merge, they generally are different at different time steps (merges of clusters introduce cluster hierarchies that are discussed further in the paper). Therefore, clustering is not absolute but depends on characteristic times of observation of the diffusive processes. The clustering algorithm, which is constructed on the basis of the definition of node similarity given above, primarily introduces a convenient reordering of nodes that makes clustering structure of the graph visible. This reordering is fully algorithmic. The cluster structure is then represented well by the clustering map - a plot of significant clusters versus diffusion time. As clusters evolve in time, there is some freedom in selecting the resulting compositions of the clusters — we expect that a well-defined cluster exceeds some minimal size and remains invariant (or approximately invariant) over some range of characteristic times.

\section{Spectral representation of the clusters}

This section gives some additional explanations and introduces an alternative interpretation of clustering based on spectral expansions. This interpretation seems to be useful for a more rigorous conceptual understanding of clustering, while the clustering algorithm of the previous section seems to be more convenient as an engineering tool. The spectral interpretation is based on the following lemma

Lemma 1 The matrix $\mathbf{P}^{n}=\left[\mathbf{p}_{1}^{n}, \mathbf{p}_{2}^{n}, \ldots, \mathbf{p}_{N}^{n}\right]$ specifying $n$-step transitional probabilities for random walk on an undirected connected graph of $N$ nodes can always be represented by the following spectral expansion

$$
\mathbf{P}^{n}=\sum_{k=1}^{N} \lambda_{k}^{n} \mathbf{v}^{k} \otimes \mathbf{a}^{k} \text { or } \mathbf{p}_{i}^{n}=\sum_{k=1}^{N} \lambda_{k}^{n} \mathbf{v}^{k} a_{i}^{k}
$$

where

$$
\mathbf{P} \mathbf{v}^{k}=\lambda_{k} \mathbf{v}^{k}, \quad \mathbf{a}^{k}=\mathbf{D}^{-1} \mathbf{v}^{k}
$$

so that the eigenvalues $\lambda_{k}$, the eigenvectors $\mathbf{v}^{k}$ and the spectral coefficients $\mathbf{a}^{k}$ are real. The eigenvectors are orthonormal

$$
\left\langle\mathbf{v}^{k}, \mathbf{v}^{j}\right\rangle=I^{k j}, \quad I^{k j}= \begin{cases}1, & k=j \\ 0, & k \neq j\end{cases}
$$

in the sense of the inner product is defined by

$$
\left\langle\mathbf{v}^{k}, \mathbf{v}^{j}\right\rangle=\left(\mathbf{v}^{k}\right)^{\mathrm{T}} \mathbf{S} \mathbf{v}^{j}=\left\langle\mathbf{v}^{j}, \mathbf{v}^{k}\right\rangle, \quad \mathbf{S}=\mathbf{S}^{\mathrm{T}}=\mathbf{D}^{-\mathbf{1}}
$$


According to definition $(13)$, the operator $\mathbf{P}$ is self-adjoint, that is $\mathbf{P}^{*}=\mathbf{P}$ where the adjoint operator $\mathbf{P}^{*}$ is defined in terms of the inner product $\langle\mathbf{x}, \mathbf{P y}\rangle=\left\langle\mathbf{P}^{*} \mathbf{x}, \mathbf{y}\right\rangle=\left\langle\mathbf{y}, \mathbf{P}^{*} \mathbf{x}\right\rangle$ with arbitrary real $\mathbf{x}$ and $\mathbf{y}$. Indeed, we may write

$$
\begin{aligned}
\langle\mathbf{x}, \mathbf{P} \mathbf{y}\rangle & =\mathbf{x}^{\mathrm{T}} \mathbf{D}^{-\mathbf{1}} \mathbf{P} \mathbf{y}=(1-\beta) \mathbf{x}^{\mathrm{T}} \mathbf{D}^{-1} \mathbf{A D}^{-1} \mathbf{y}+\beta \mathbf{x}^{\mathrm{T}} \mathbf{D}^{-1} \mathbf{y} \\
& =(1-\beta) \mathbf{y}^{\mathrm{T}} \mathbf{D}^{-1} \mathbf{A}^{\mathrm{T}} \mathbf{D}^{-1} \mathbf{x}+\beta \mathbf{y}^{\mathrm{T}} \mathbf{D}^{-1} \mathbf{x}=\mathbf{x}^{\mathrm{T}} \mathbf{D}^{-\mathbf{1}} \mathbf{P} \mathbf{y}=\langle\mathbf{y}, \mathbf{P} \mathbf{x}\rangle
\end{aligned}
$$

for any $\mathbf{x}$ and $\mathbf{y}$ since $\mathbf{A}$ is symmetric $\left(\mathbf{A}^{\mathrm{T}}=\mathbf{A}\right)$ and $\mathbf{D}$ is diagonal. Self-adjoint compact operators are subject to the Hilbert-Schmidt theorem (being defined in a Euclidean space of finite dimension, the operator $\mathbf{P}$ is always compact - see Kolmogorov \& Fomin (1957)). Hence, according to this theorem, the eigenvalues $\lambda_{k}$ are real $\left(\left|\lambda_{k}\right|=\lambda_{k}\right)$ and eigenvectors $\mathbf{v}^{k}$ can be chosen real and orthonormal (in the sense of the inner product defined by (13) but not in the sense of the dot product used in (7)). Finally we note that operation $a^{k}=\left\langle\mathbf{v}^{k}, \mathbf{y}\right\rangle=\left(\mathbf{v}^{k}\right)^{\mathrm{T}} \mathbf{D}^{-1} \mathbf{y}$ determines coefficients for spectral expansion $\mathbf{y}=\mathbf{\Sigma}_{k} \mathbf{v}^{k} a^{k}$ of an arbitrary vector $\mathbf{y}$ in the basis of $\mathbf{v}^{k}$. Hence $\mathbf{P y}=\boldsymbol{\Sigma}_{k} \lambda_{k} \mathbf{v}^{k} a^{k}$. Since this basis is complete, the transition operator $\mathbf{P}$ can be expressed in terms of the outer product by

$$
\mathbf{P}=\sum_{k=1}^{N} \lambda_{k}\left(\mathbf{v}^{k} \otimes \mathbf{v}^{k}\right) \mathbf{D}^{-1}=\sum_{k=1}^{N} \lambda_{k} \mathbf{v}^{k}\left(\mathbf{a}^{k}\right)^{\mathrm{T}}
$$

Applying operator $\mathbf{P} n$ times, we obtain 10 . Expansion 10 is unique as long as the eigenvalues are not repeated.

It is easy to see that matrices $\mathbf{T}$ and $P$ have the same set of eigenvectors $\mathbf{T} \mathbf{v}^{k}=\mu_{k} \mathbf{v}^{k}$ and $\mathbf{P} \mathbf{v}^{k}=\lambda_{k} \mathbf{v}^{k}$ but shifted eigenvalues $\lambda_{k}=(1-\beta) \mu_{k}+\beta$. Since the absolute values of eigenvalues of $\mathbf{T}$ are bounded by unity $\left|\mu_{k}\right| \leq 1$ (the matrix $\mathbf{T}$ must preserve the overall probability $\Sigma_{i} p_{i}=1$ ), the choice of $\beta=1 / 2$ enforces non-negativeness of the eigenvalues $0 \leq \lambda_{k} \leq 1$. This underpins predominately monotonic convergence $\mathbf{p}_{i}^{n} \rightarrow \mathbf{p}^{\circ}$ as $n \rightarrow \infty$ (as it is shown above, eigenvalues $\lambda_{k}$ must be real $\lambda_{k}=\left|\lambda_{k}\right|$ ). Since the graph is presumed to be connected, there exists $n_{0}$ so that all transitional probabilities are strictly positive $P_{j i}^{n}>0$ for any $n \geq n_{0}$. Hence, the transition matrix is subject to the conditions of the Perron-Frobenius theorem (see Gantmakher, 1959) so that its largest eigenvalue is positive, distinct and must be $\lambda_{1}=1$ to preserve the overall probability. Hence, without loss of generality, we presume in the rest of the paper that the eigenvalues are ordered

$$
1=\lambda_{1}>\lambda_{2} \geq \ldots \geq \lambda_{N} \geq 0
$$

Any quantities related to a selected group of the largest lambdas (e.g. $\left.\lambda_{1}, \ldots, \lambda_{k}, \quad k<N\right)$ are be referred to as "leading".

We can define our understanding of clusters in terms of the spectral expansions specified by $(10)$. In its spectral form, the clustering assumption becomes

$$
a_{i}^{k}=a_{\mathfrak{C}}^{k} \text { for } k \leq m(\mathfrak{C}) \text { and } i \in \mathfrak{C}
$$

that is for any node $i$ that belongs to cluster $\mathfrak{C}$ there exist such $m$ dependent on $\mathfrak{C}$ that for any $k \leq m$ the spectral coefficient are independent of $i$. The coefficients $a_{\mathfrak{C}}^{1}, \ldots, a_{\mathfrak{C}}^{m}$ are thus spectral characteristics of the cluster $\mathfrak{C}$ and would be different for a different cluster. Hence, the powers of the transition operator can be represented by

$$
\mathbf{p}_{i}^{n}=\mathbf{p}_{\mathfrak{C}}^{n}+\mathbf{g}_{i}^{n}(\mathfrak{C}), \quad i \in \mathfrak{C}
$$

where

$$
\mathbf{p}_{\mathfrak{C}}^{n}=\sum_{k=1}^{m} \lambda_{k}^{n} \mathbf{v}^{k} a_{\mathfrak{C}}^{k}, \quad \mathbf{g}_{i}^{n}(\mathfrak{C})=\sum_{k=m+1}^{N} \lambda_{k}^{n} \mathbf{v}^{k} a_{i}^{k} \sim O\left(\lambda_{m+1}^{n}\right)
$$

We note that, according to this definition, all nodes belong to the overall graph cluster $\mathfrak{G}$ that has $m=1$ and involves all nodes of the graph: $a_{i}^{1}=a_{\mathfrak{G}}^{1}$ for all $i=1, \ldots, N$. Each cluster $\mathfrak{C}$ is associated with the characteristic time

$$
t_{\mathfrak{C}}=\frac{1}{\left|\ln \lambda_{m+1}\right|}
$$

so that $\mathbf{g}_{i}^{n}(\mathfrak{C})$ is exponentially small for $t \gg t_{\mathfrak{C}}$, no larger than $\sim \exp \left(-t / t_{\mathfrak{C}}\right)$. We denote $t=n$ to clearly outline the time dependence. A cluster $\mathfrak{C}$ should appear well before the stationary distributions are established to be distinguishable from the overall cluster $\mathfrak{G}$. Hence it is expected that $t_{\mathfrak{C}} \ll t_{\mathfrak{G}}=1 /\left|\ln \lambda_{2}\right|$ 
for any cluster $\mathfrak{C}$ distinguishable from $\mathfrak{G}$. Here, $t_{\mathfrak{G}}$ is the characteristic time of achieving steady-state distributions in the whole network.

In practice, however, condition (16) is not satisfied exactly for any $m>1$. Only when $m=1$, all of $a_{1}^{1}, \ldots, a_{N}^{1}$ are exactly the same to specify the stationary solution. This implies that equation (17) needs to be corrected for deviations of $a_{i}^{k}$ from $a_{\mathfrak{C}}^{k}$, which nevertheless are expected to be small. To reflect this, condition 16 is replaced by

$$
\left|a_{i}^{k}-a_{\mathfrak{C}}^{k}\right| \sim \varepsilon \ll 1 \text { for } k \leq m(\mathfrak{C}) \text { and } i \in \mathfrak{C}
$$

We therefore obtain:

$$
\mathbf{p}_{i}^{n}=\mathbf{p}_{\mathfrak{C}}^{n}+\mathbf{g}_{i}^{n}(\mathfrak{C})+\mathbf{h}_{i}^{n}(\mathfrak{C}), \quad i \in \mathfrak{C}
$$

where

$$
\mathbf{h}_{i}^{n}(\mathfrak{C})=\sum_{k=2}^{m} \lambda_{k}^{n} \mathbf{v}^{k}\left(a_{i}^{k}-a_{\mathfrak{C}}^{k}\right) \sim \varepsilon O\left(\lambda_{2}^{n}\right)=\varepsilon O\left(\exp \left(-\frac{t}{t_{\mathfrak{G}}}\right)\right)
$$

reflects spectral imperfections in representation of the clusters.

Our analysis becomes more transparent if the following interpretation of the similarity criterion is used:

$$
R_{i j}^{n}=\frac{\left\langle\mathbf{p}_{i}^{n}, \mathbf{p}_{j}^{n}\right\rangle}{\left\|\mathbf{p}_{i}^{n}\right\|\left\|\mathbf{p}_{j}^{n}\right\|}, \quad R_{i j}^{n} \geq 1-\delta \Longrightarrow \mathbf{p}_{i}^{n} \cong \mathbf{p}_{j}^{n}
$$

where $\|\mathbf{x}\| \equiv\langle\mathbf{x}, \mathbf{x}\rangle^{-1 / 2}, \quad\langle\mathbf{x}, \mathbf{y}\rangle=\mathbf{x}^{\mathrm{T}} \mathbf{S y}$ for any $\mathbf{x}$ and $\mathbf{y}$. If $\mathbf{p}_{i}^{n}=\mathbf{p}_{\mathfrak{C}}^{n}+f_{i}^{n}$ then we can expand assuming that variations $f_{i}^{n}$ are small

$$
R_{i j}^{n}=1-\frac{1}{2} \frac{\left\langle\mathbf{f}_{i}^{n}-\mathbf{f}_{j}^{n}, \mathbf{f}_{i}^{n}-\mathbf{f}_{j}^{n}\right\rangle}{\left\langle\mathbf{p}_{\mathfrak{C}}^{n}, \mathbf{p}_{\mathfrak{C}}^{n}\right\rangle}+\frac{1}{2} \frac{\left\langle\mathbf{p}_{\mathfrak{C}}^{n}, \mathbf{f}_{i}^{n}-\mathbf{f}_{j}^{n}\right\rangle^{2}}{\left\langle\mathbf{p}_{\mathfrak{C}}^{n}, \mathbf{p}_{\mathfrak{C}}^{n}\right\rangle^{2}}+O\left(\left\|\mathbf{f}_{i}^{n}-\mathbf{f}_{j}^{n}\right\|^{3}\right)
$$

To avoid unnecessary complexities, we put $\mathbf{S}=\mathbf{D}^{-1}$ ensuring orthogonality of $\mathbf{g}_{i}^{n}(\mathfrak{C})$ and $\mathbf{h}_{i}^{n}(\mathfrak{C})$. That is $\left\langle\mathbf{g}_{i}^{n}, \mathbf{h}_{j}^{n}\right\rangle=0$ for any $i, j \in \mathfrak{C}$ and also $\left\langle\mathbf{g}_{i}^{n}, \mathbf{p}_{\mathfrak{C}}^{n}\right\rangle=0$ for any $i \in \mathfrak{C}$. Substitution of $\mathbf{f}_{i}^{n}=\mathbf{g}_{i}^{n}+\mathbf{h}_{i}^{n}$ into 24) yields

$$
\begin{aligned}
R_{i j}^{n} & =1-\frac{1}{2} \frac{\left\|\mathbf{g}_{i}^{n}-\mathbf{g}_{j}^{n}\right\|^{2}+\left\|\mathbf{h}_{i}^{n}-\mathbf{h}_{j}^{n}\right\|^{2}}{\left\|\mathbf{p}_{\mathfrak{C}}^{n}\right\|^{2}}+\frac{1}{2} \frac{\left\langle\mathbf{p}_{\mathfrak{C}}^{n}, \mathbf{h}_{i}^{n}-\mathbf{h}_{j}^{n}\right\rangle^{2}}{\left\|\mathbf{p}_{\mathfrak{C}}^{n}\right\|^{4}}+\ldots \\
& =1-O \underbrace{\left(\exp \left(-2 \frac{t}{t_{\mathfrak{C}}}\right)\right)}_{\sim \lambda_{m+1}^{2 n}}-\varepsilon^{2} O \underbrace{\left(\exp \left(-2 \frac{t}{t_{\mathfrak{G}}}\right)\right)}_{\sim \lambda_{2}^{2 n}}
\end{aligned}
$$

for $i, j \in \mathfrak{C}$ and $t \rightarrow \infty$. This representation imposes restrictions on the choice of the threshold $\delta$ in (7). Indeed, on one hand we wish to set $\delta$ sufficiently small to ensure accurate representation of the clusters. On the other hand, if $\lambda_{m+1}^{n} \sim \delta$ then $\lambda_{2}^{n} \gg \lambda_{m+1}^{n}$ and, generally, $\lambda_{2}^{n}$ should be treated as being of the order of unity since, as discussed previously, $t_{\mathfrak{G}} \gg t_{\mathfrak{C}}$. Hence, we should assume that the last term is $\sim \varepsilon^{2}$ at times $t \gtrsim t_{\mathfrak{C}}$ when cluster $\mathfrak{C}$ is being detected and select the threshold $\delta$ within the range

$$
1 \gg \delta \gg \varepsilon^{2}
$$

to avoid interference of the spectral imperfections with the similarity criterion given in (23).

The spectral understanding of clustering can be summarised in the following proposition

Proposition 2 A cluster $\mathfrak{C}$ is a group of nodes that have the same or similar values of $m$ leading spectral coefficients, i.e. $a_{i}^{k} \approx a_{j}^{k}$ where $i, j \in \mathfrak{C}, k=1,2, \ldots, m$ and $\mathbf{a}^{k}=\mathbf{D}^{-1} \mathbf{v}^{k}$. Different clusters have different spectral coefficients and, generally, different values of $m$. Each cluster $\mathfrak{C}$ is associated with a certain characteristic diffusion time given by $t_{\mathfrak{C}}=\left|\ln \lambda_{m+1}\right|^{-1}$.

Finally, it is useful to stress the relation between the temporal and spectral properties used to define clusters: 
Proposition 3 If $m$ leading spectral coefficients are the same $a_{i}^{k}=a_{j}^{k}$ for two nodes $i$ and $j$ and for $k=1, \ldots, m$, then $R_{i j}^{n}=1-O\left(\lambda_{m+1}^{2 n}\right)$ as $n \rightarrow \infty$. If $R_{i j}^{n}=1-O\left(\omega^{2 n}\right)$ as $n \rightarrow \infty$, for two nodes $i$ and $j$, and for any fixed value $0<\omega<1$, then the leading spectral coefficients must be the same $a_{i}^{k}=a_{j}^{k}$ for any $k$ that $\lambda_{k}>\omega$.

The first part of the statement immediately follows from the spectral expansion in Lemma 1 and is obvious. The second part can be easily proven by assuming that $a_{i}^{k} \neq a_{j}^{k}$ and finding that the result $R_{i j}^{n}=1-O\left(\lambda_{k}^{2 n}\right)$ contradicts the condition $R_{i j}^{n}=1-O\left(\omega^{2 n}\right)$ in the statement when $\lambda_{k}>\omega$. There is another possibility of $a_{i}^{k}=c a_{j}^{k}$ with constant $c \neq 1$ and $k=1, \ldots, m$ where $\lambda_{m+1} \leq \omega$, which is compliant with $R_{i j}^{n}=1-O\left(\omega^{2 n}\right)$. This case, however, is inconsistent with the stationary distribution requiring $a_{i}^{1}=a_{j}^{1}$.

\section{Clustering of industrial network}

The procedure specified in Section 3 is used here with $\beta=1 / 2$ in $[5]$ and $\delta=10^{-2}$ in $(8)$. As noted in Section 3 . the binary relation of cluster similarity is subjected to transitive closure to avoid dependence of clusters on initial ordering of the nodes. Unless otherwise stated, the nodes are preordered using the Fiedler vector. The graph of industrial product connections was constructed following the procedure suggested by Hidalgo et al. (2007). The data are taken from the United Nation Comtrade website using SITC2 classification at 4-digit level. This specifies 770 categories of products traded by 153 countries. The graph with $N=770$ nodes is then constructed by Kruskal's algorithm followed by adding another 1000 connections that have highest proximities. The details can be found in thesis by Bouet (2018). All these steps and parameters are consistent with those selected by Hidalgo et al. (2007) and Hausmann et al. (2014). The results presented below are generally stable with respects to the selection of the parameters.

A similar group of nine primary clusters tends to appear irrespective of the variations in the clustering procedure, although some details and the final ordering may vary. The cluster structure is shown by clustering maps in Figure 2. Primary clusters, which have the shortest characteristic times, tend to merge into secondary clusters that have longer characteristic times and, after several rounds of merges, form the overall cluster $\mathfrak{G}$ that covers all of the nodes. The two maps shown correspond to different initial (and final) orderings, according to SITC classification on the left and using the Fiedler vector on the right. Yet the clusters, which are indicated by numbers and red lines, are exactly the same. Ordering of elements on the maps does not change with time: each of the clustering maps uses the corresponding final ordering produced by the clustering algorithm. Figure 2 also indicates the characteristic time scales of cluster formation, growth and merging. It can be seen that, in general, cluster compositions evolve with time and thus can be selected differently (in Figure 2, out selection is indicated by vertical lines). We nevertheless expect that a well-defined cluster exists in a fixed or slightly changing boundaries over some range of characteristic times.

The determined primary clusters are briefly described in Table 1. Many of the clusters (at least 3 "mechanical" clusters) are associated with machinery. Two of these clusters (Machinery-2 and Machinery3) merge early to form the secondary Machinery cluster. Machinery-3 is more related to construction than Machinery-1. The third machinery cluster (Machinery-1) seems to be more closely associated with general chemical industry (Chemicals-1). It is interesting that the second chemical cluster (Chemicals-2), which is more specialised and less related to Machinery than the first chemical cluster, takes longer to appear. Food industry (Food) and building materials industry (Construction) appear to be closely related through. Garments form a very distinctive and quite independent cluster involving many related products. Mining and resources hardly form an independent cluster and are distributed between other clusters.

The clustering algorithm used in the present work forms the same clusters irrespective of the initial ordering. These clusters are formed not due to logical ordering of products by SITC classification (and, as discussed below, are initially reordered by the Fiedler vector) but through connections between industry products. Figure 3 displays the same adjacency matrix A with different ordering of the nodes: a) according to SITC classification, b) using the Fiedler vector, c) using clustering algorithm on original SITC ordering and d) using clustering algorithm after pre-ordering by the Fiedler vector. The clustering algorithm of the present work identifies some "fast" clusters that are missing by the sorting using the Fiedler vector since the latter pertains to the divisions associated with the slowest relaxation to the steady-state distribution.

Some of the products may be found in clusters that are seemingly not related or related by practice rather than by a common origin or a conceptual link. For example many packaging products can be found not in Chemicals but in the Food cluster, where these products are predominately used. Various resources and materials can often be found in industrial clusters that use them. Figure 4 compares SITC ordering 
of the products with ordering achieved by the clustering algorithm. The colour code corresponds to 1-digit SITC codes. The nine primary clusters are indicated by the vertical bars. The correspondence between the identified clusters and 1-digit codes is apparent. While good ordering is important for visualisation, Figures 4 and 5 use initial odering ordering by the Fiedler vector and not by the SITC codes. This is to make sure that the clustering algorithm is not aware about the relationships between products, which is indicated by the standard ordering of the SITC codes. Effectively, the clustering algorithm cannot benefit from knowing the SITC codes and has to introduce its own classification of the products from properties of the industrial network. The classification by the algorithm does not match the SITC codes exactly but appears to correlate with them. This correlation can be improved by using 2-digit SITC codes and mapping them onto 8 categories that do not coincide with the 1-digit SITC codes. The SITC codes reflect formal classification that is traditionally used in trade statistics but does not necessarily correspond to the role that these products play in the real world. Figure 5 demonstrates that this new refined coding improves characterisation of the clusters. As shown in Figure 6, the refined coding is achieved in two stages: first, mapping is performed on the basis of the 1-digit codes as indicated by the thick arrows and, second, some of the 2-digit categories of products are remapped according to our understanding of their roles as shown by the thin arrows.

Finally, Figure 7 demonstrates spectral representation for selected clusters. Each subfigure characterises a single cluster and plots 20 lines with each line corresponding to a distinct node from the cluster. The lines are not similar in the top subfigure - this subfigure does not correspond to any cluster and is shown for comparison. The lines of the bottom subfigures match much better than those of the top subfigures. The effective value of $m$ increases and the corresponding characteristic time $t_{\mathfrak{C}}$ decreases from top to bottom. Hence, lower subfigures correspond to faster forming clusters with shorter characteristic times. This is consistent with cluster properties shown in Figure 2. We also note that the first 5-10 modes are similar for the clusters Chemicals-2 and Electrical but different from the corresponding modes determined for the cluster Garment. This is expected since clusters Chemicals-2 and Electrical merge to form a secondary cluster.

\section{Discussion of the findings}

Absence of an autonomous cluster representing resources and mining seems puzzling. Some of the simulations produced a cluster resembling mining, but its detection was not reliable and such sporadically appearing clusters are not shown in the present work (detection of clusters that have long characteristic diffusion times cannot be reliable). Two factors can be responsible for this behaviour. The first is strong linking of resources with the industries that use these resources; the second is that mining and resources are subject to geological constraints and thus may be strongly affected by non-economic factors, which are not considered in the theory due to Hidalgo et al. (2007).

The time dependency of the clustering algorithm is essential for our analysis. This time, however, reflects only the rate of diffusion of innovation and should not be confused with the real physical time. While the specific shapes of the detected clusters may be to some extent unexpected, the main findings of this work tend to agree with what is generally known about industrial development (Hidalgo et al., 2007). This confirms the approach to clustering implemented in the present work. Industrial evolution forms clusters of industries that tend to grow jointly in dynamic connection with each other. Developing industries associated with clusters that have more cohesive structure and smaller characteristic diffusion times is easier since these clusters require less diverse resources and connections. The garment industries are the fastest to develop, followed by agroindustry, construction and machinery. Industries producing complex chemicals and electronics tend to develop slower. The chemical industry has two quite independent clusters: traditional chemistry (Chemicals-1), which develops faster, and advanced chemistry (Chemicals-2), whose development takes a longer time. This is consistent with commonly known economic trends: many developing countries start from garment and agricultural industries and develop heavier industries at later stages. In some newlydeveloped economies, electronics may be one of the early additions to the industrial mix but this seems to be the effect of government intervention.

The present consideration is based on modern trade data, which cannot accurately reflect links between industries that existed many decades ago. Yet, there is something common present in the two and half centuries-long industrial development of the world. The five Kondratiev waves of economic growth, which were recognised by Kondratiev, conceptualised by Schumpeter and documented by Freeman, were re-evaluated by Perez as surges of technological innovation. These surges are initiated by technological 
breakthroughs and drive subsequent waves of economic growth. These technological surges, which are schematically presented in Figure 8 are roughly consistent with the diffusional speeds of cluster formation obtained in the present work. The British revolution in textile production is followed by surges of innovation in machinery and construction, and only then by radical changes in chemistry and electronics. The data characterising the present state of industrial production cannot possibly reproduce the history of industrial development, but it seems that these data reflect some technological fundamentals that tend to persist over time. Modern industry is still not homogeneous and forms clusters of related products. Therefore, we can and, in fact, should expect a forthcoming sixth surge in industrial development, which seems to be emerging and is likely to be related to communications, transport, automation, AI, commercial space exploration, advanced materials and medicine, new sources of energy as well as artificial intelligence, advanced knowledge and progressive education.

\section{Conclusions}

This work suggests an alternative implementation of the ideas, which were introduced by Lambiotte \& Barahona (2009), to examine a hierarchy of network communities by a random walk. This implementation involves a resorting algorithm combined with identification of clusters on the basis of similarity of the transitional properties of random walks originated at the nodes forming a cluster. Different diffusion times correspond to clusters of different levels. In spectral representation, the same property is reflected in similarity of the leading spectral coefficients. While this work endeavours to accurately define clusters, we must note that, by their nature, clustering properties are not exact and always leave some freedom in defining what similarity means in exact terms. Our interpretation of clusters reflects physical understanding of industrial links introduced and evaluated by Hidalgo et al. (2007) and Hausmann et al. (2014).

The clusters considered here involve a characteristic time scale as one of the properties of every cluster. The presence of a time scale in the analysis is consistent with the understanding of diffusion of industrial technology introduced by Hidalgo et al. (2007). This understanding links the rate of innovation to the rate of diffusion of randomly walking particles on a graph representing connections between industrial products. The present analysis identifies around a dozen clusters of industrial products and is consistent with the notion that modern industry forms well-connected technological clusters. Conceptual agreement with known historical trends in evolution of industries supports the thesis about the leading role of technological progress in long-term economic changes. 


\section{References}

Aldous, D. \& Fill, J. (2014) Reversible Markov chains and random walks on graphs. unfinished monograph, http://www.stat.berkeley.edu/ aldous/RWG/book.html.

Bassett, D. S., Porter, M. A., Wymbs, N. F., Grafton, S. T., Carlson, J. M. \& Mucha, P. J. (2013) Robust detection of dynamic community structure in networks. Chaos: An Interdisciplinary Journal of Nonlinear Science, 23(1).

Bouet, V. (2018) Modelling of complex competitive systems applied to the study of technical revolutions. M.Eng.Sci. Thesis, The University of Queensland, SoMME.

Delvenne, J.-C., Yaliraki, S. N. \& Barahona, M. (2010) Stability of graph communities across time scales. Proceedings of the National Academy of Sciences, 107(29).

Fortunato, S. (2009) Community detection in graphs. Physics Reports, 486(3-5).

Fortunato, S. \& Hric, D. (2016) Community detection in networks: A user guide. Physics Reports, 659(C), $1-44$.

Freeman, C. \& Soete, L. (1997) Economics of Industrial Innovation. Routledge, London.

Gantmakher, F. R. (1959) The theory of matrices. Chelsea, New York.

Grimmett, G. (2018) Probability on Graphs. Cambridge University Press, Cambridge.

Grinstead, C. M. \& Snell, J. L. (1997) Introduction to probability. Open textbook library. American Mathematical Society, Providence, RI, 2nd rev. ed. / edition.

Hausmann, R., Hidalgo, C. A., Bustos, S., Coscia, M., Simoes, A. \& Yildirim, M. A. (2014) The Atlas of Economic Complexity : Mapping Paths to Prosperity. MIT Press, Cambridge.

Hidalgo, C. A., Klinger, B., Barabsi, A.-L. \& Hausmann, R. (2007) The product space conditions the development of nations. Science, $\mathbf{3 1 7}(5837)$.

Holme, P. (2015) Modern temporal network theory: a colloquium. The European Physical Journal B - Condensed Matter and Complex Systems, 88(9).

Holme, P. \& Saramaki, J. (2012) Temporal networks. Physics Reports, 519(2).

Jeub, L. G. S., Balachandran, P., Porter, M. A., Mucha, P. J. \& Mahoney, M. W. (2015) Think locally, act locally: detection of small, medium-sized, and large communities in large networks.. Physical review. E, Statistical, nonlinear, and soft matter physics, 91(1), 012821-012821.

Kolmogorov, A. N. \& Fomin, S. V. (1957) Elements of the theory of functions and functional analysis. Gaylock Press, Rochester, N.Y.

Lambiotte, R. \& Barahona, M. (2009) Laplacian Dynamics and Multiscale Modular Structure in Networks. arXiv.org.

Lambiotte, R., Delvenne, J.-C. \& Barahona, M. (2015) Random Walks, Markov Processes and the Multiscale Modular Organization of Complex Networks. IEEE Transactions on Network Science and Engineering, 1(2), $76-90$.

Lancichinetti, A., Fortunato, S. \& Kertsz, J. (2009) Detecting the overlapping and hierarchical community structure in complex networks. New Journal of Physics, 11(3).

Lyzinski, V., Tang, M., Athreya, A., Park, Y. \& Priebe, C. E. (2017) Community Detection and Classification in Hierarchical Stochastic Blockmodels. Network Science and Engineering, IEEE Transactions, 4(1), 13-26.

Masuda, N. \& Lambiotte, R. (2016) A guide to temporal networks. World Scientific Publishing Europe Ltd., London.

Masuda, N., Porter, M. A. \& Lambiotte, R. (2017) Random walks and diffusion on networks. Physics Reports, 716-717(C), 1-58.

Mucha, P. J., Richardson, T., Macon, K., Porter, M. A. \& Onnela, J.-P. (2010) Community structure in time-dependent, multiscale, and multiplex networks. Science, 328(5980).

Perez, C. (2006) Respecialisation and the Deployment of the ICT Paradigm: An Essay on the Present Challenges of Globalization. In Compano, R., Pascu, C., Bianchi, A., Burgelman, J.-C., Barrios, S., Ulbrich, M. \& Maghiros, I., editors, The Future of the Information Society in Europe: Contributions to the Debate, volume EUR 22353, pages 33-66, Seville, Spain. European Commission, Directorate General Joint Research Center.

Porter, M. A., Onnela, J.-P. \& Mucha, P. J. (2009) Communities in Networks. Notices of the American Mathematical Society, 56(9), 1082-1097, 1164-1166.

Rosvall, M., Esquivel, A. V., Lancichinetti, A., West, J. D. \& Lambiotte, R. (2014) Memory in network flows and its effects on spreading dynamics and community detection. Nature Communications, 5(1). 
Salnikov, V., Schaub, M. T. \& Lambiotte, R. (2016) Using higher-order Markov models to reveal flow-based communities in networks. Scientific Reports, 6(1).

Schaub, M. T., Delvenne, J.-C., Yaliraki, S. N. \& Barahona, M. (2012) Markov Dynamics as a Zooming Lens for Multiscale Community Detection: Non Clique-Like Communities and the Field-of-View Limit (Non Clique-Like Communities). PLoS ONE, 7(2).

Schumpeter, J. A. (1947) Capitalism, socialism and democracy. Allen and Unwin, London, 2nd rev. ed.. edition. 


\begin{tabular}{|c|c|c|}
\hline No & $\begin{array}{l}\text { Cluster } \\
\text { Label }\end{array}$ & Main products associated with the cluster \\
\hline 1 & Chemicals-1 & $\begin{array}{l}\text { Common chemicals (organic and non-organic), some processed materials } \\
\text { and related machinery }\end{array}$ \\
\hline 2 & Machinery-1 & $\begin{array}{l}\text { Mostly machinery, often specialised, some products related to chemicals, } \\
\text { paper and food processing }\end{array}$ \\
\hline 3 & Machinery-2 & $\begin{array}{l}\text { General machinery and equipment (a few are related to transport, en- } \\
\text { ergy and military), measuring and controlling devices, some supplies and } \\
\text { materials. }\end{array}$ \\
\hline 4 & Machinery-3 & $\begin{array}{l}\text { Machinery and related materials, a few are related to construction and } \\
\text { agriculture }\end{array}$ \\
\hline 5 & Construction & $\begin{array}{l}\text { Construction materials and equipment, some agricultural and household } \\
\text { products, furniture }\end{array}$ \\
\hline 6 & Food & Foods, drinks, tobacco, feeds and other agricultural products, packaging \\
\hline 7 & Chemicals-2 & $\begin{array}{l}\text { Mixture of chemicals, gases and materials (including industrial and for a } \\
\text { special use, such as art, image \& photo products), chemical and nuclear } \\
\text { reactors }\end{array}$ \\
\hline 8 & Electrical & $\begin{array}{l}\text { Electrical machinery and equipment, electronics, telecommunications, dig- } \\
\text { ital processors, circuits and controls, photo, optical and other related } \\
\text { equipment, some related materials }\end{array}$ \\
\hline 9 & Garment & $\begin{array}{l}\text { Closing, footwear, personal items, some fabrics and related processed ma- } \\
\text { terials }\end{array}$ \\
\hline
\end{tabular}

Table 1. Brief specification of the primary industrial clusters. 


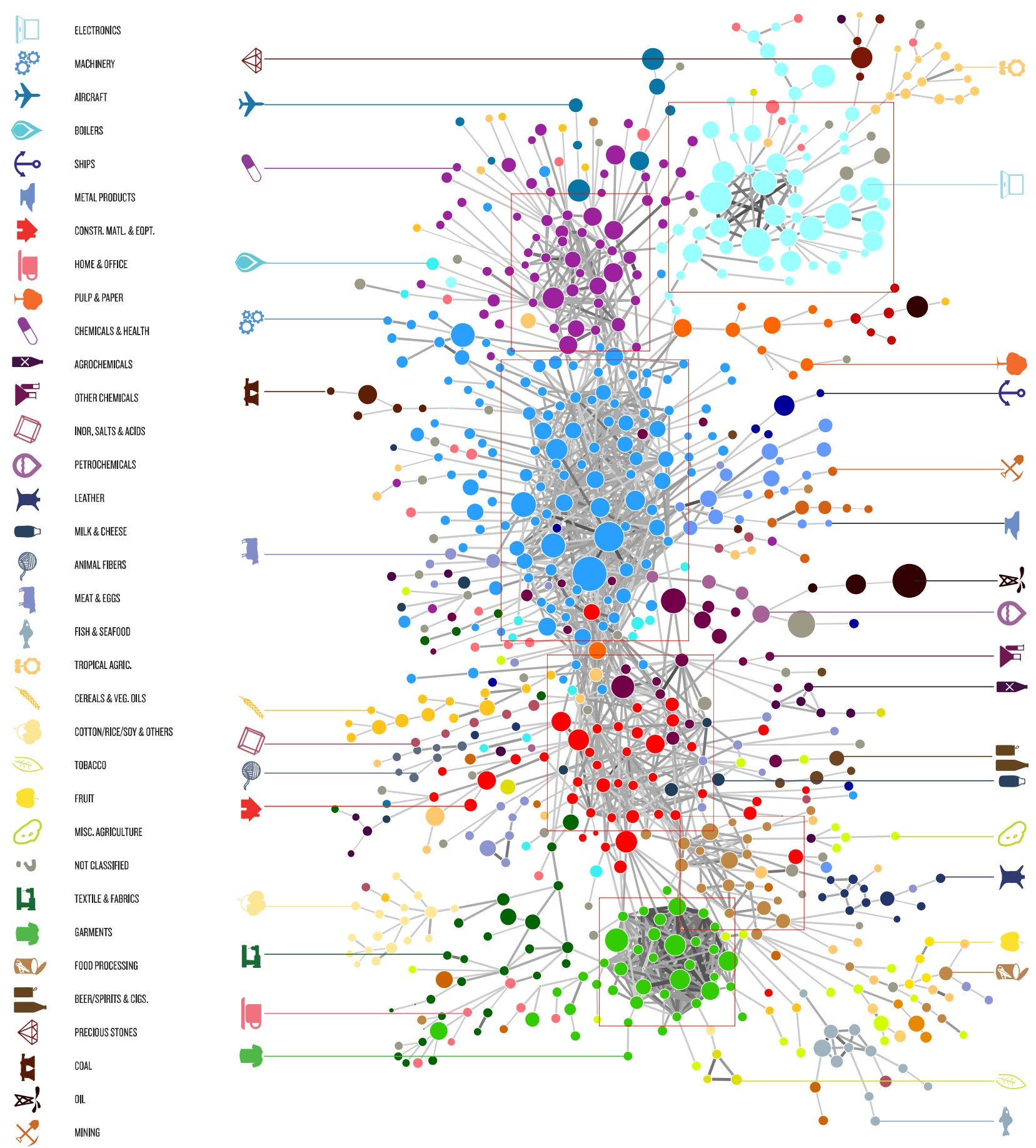

Fig. 1 Connections between industrial producs due to Hausmann et al. (2014) (thanks: Cesar A. Hidalgo). The rectangles outline the core segments of the visually apparent clusters. 

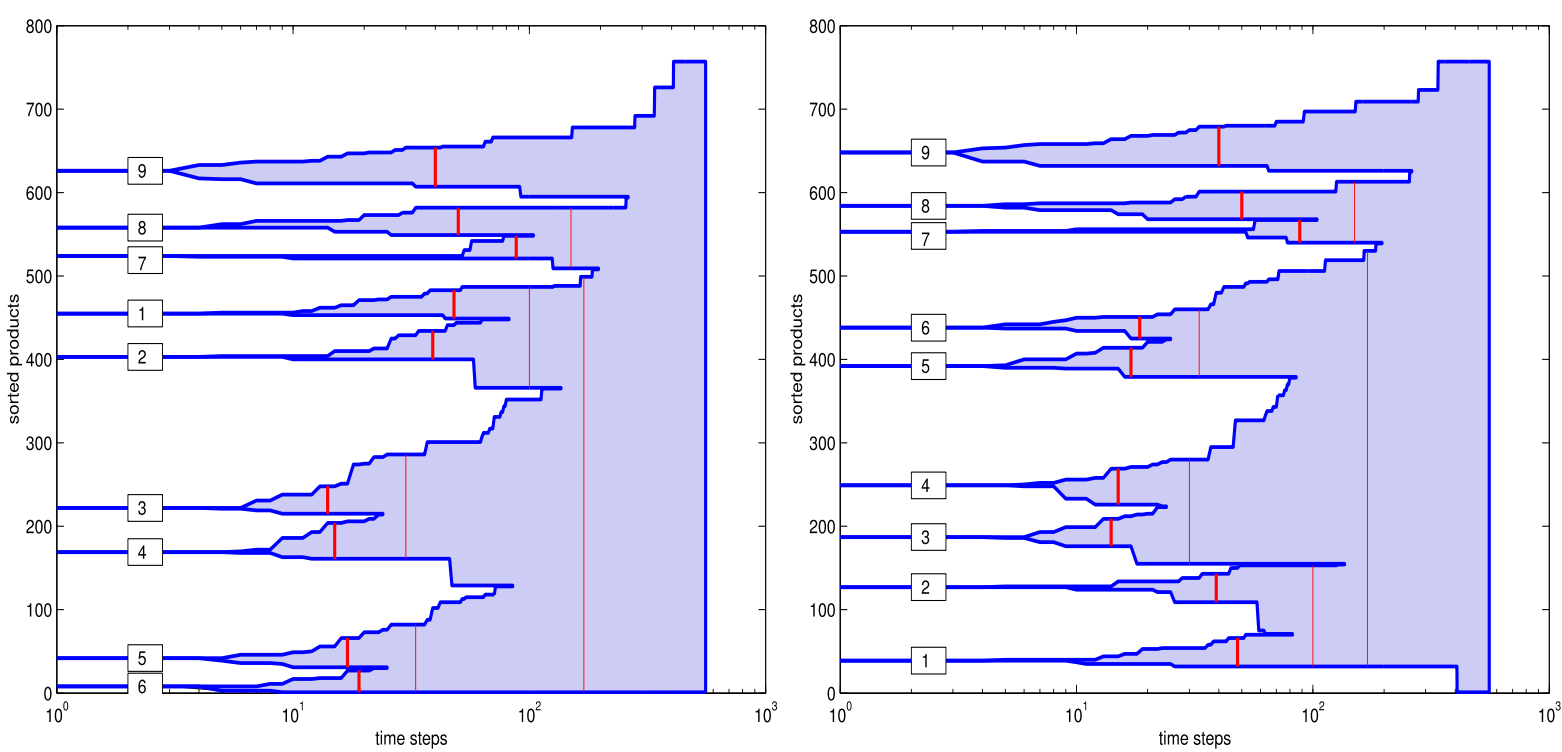

Fig. 2 Clustering map: extent of clusters of industrial products versus time step. The vertical lines show locations where the identified clusters are sampled (to be shown in the other figures; thick lines correspond to primary clusters). The initial ordering is according to SITC classification (left) or using the Fiedler vector (right). 

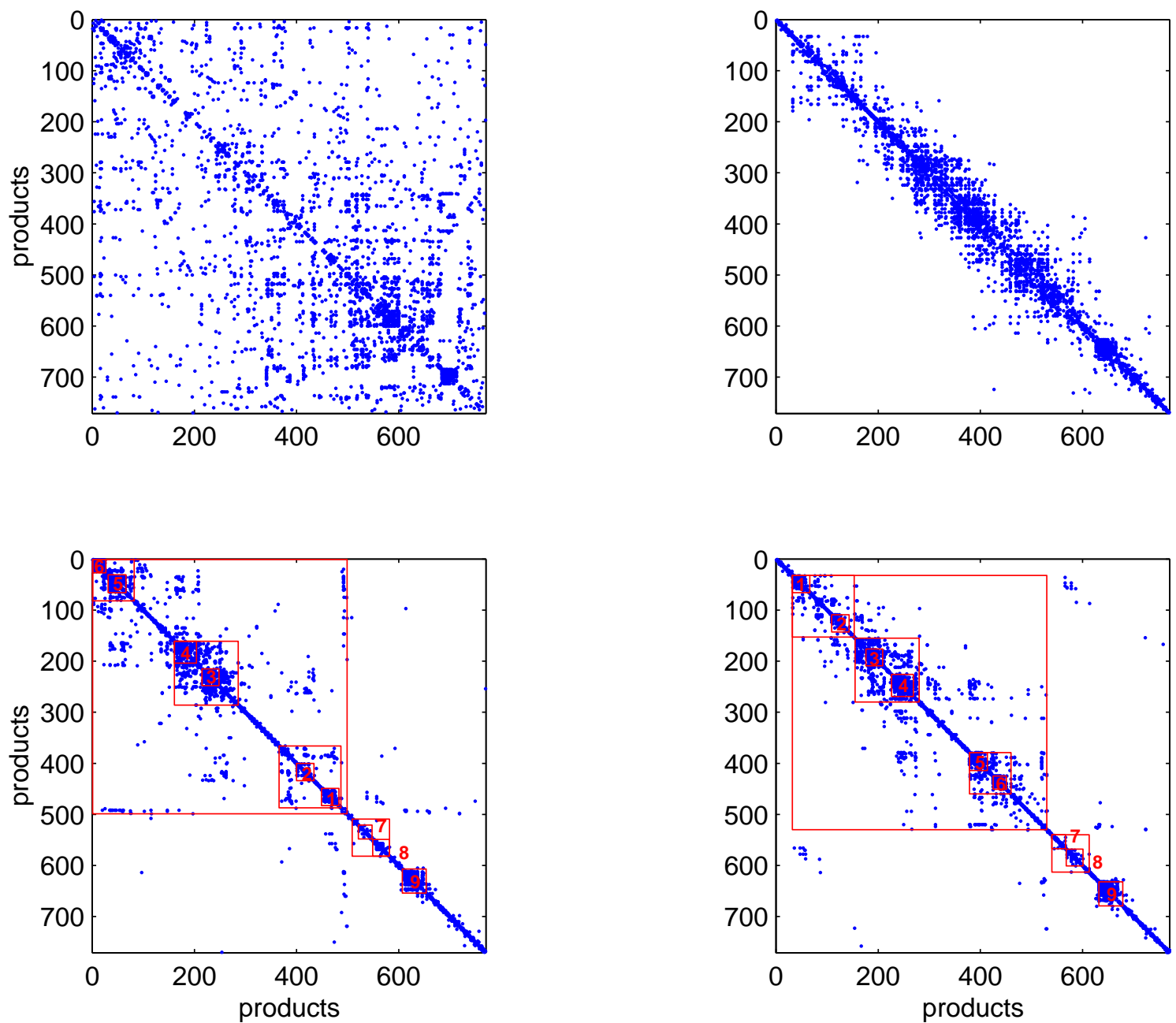

Fig. 3 Adjacency matrix shown with different sorting. Top row: no clustering, botom row: sorting by clustering algorithm. Left column: (pre-)sorting according to SITC codes, right column: (pre-)sorting by the Fiedler vector. The clusters identified in Figure ?? are indicated by red squares. The numbers of the nine primary clusters are also shown. These clusters are the same in the two bottom figures and selected as shown in Figure 2. 


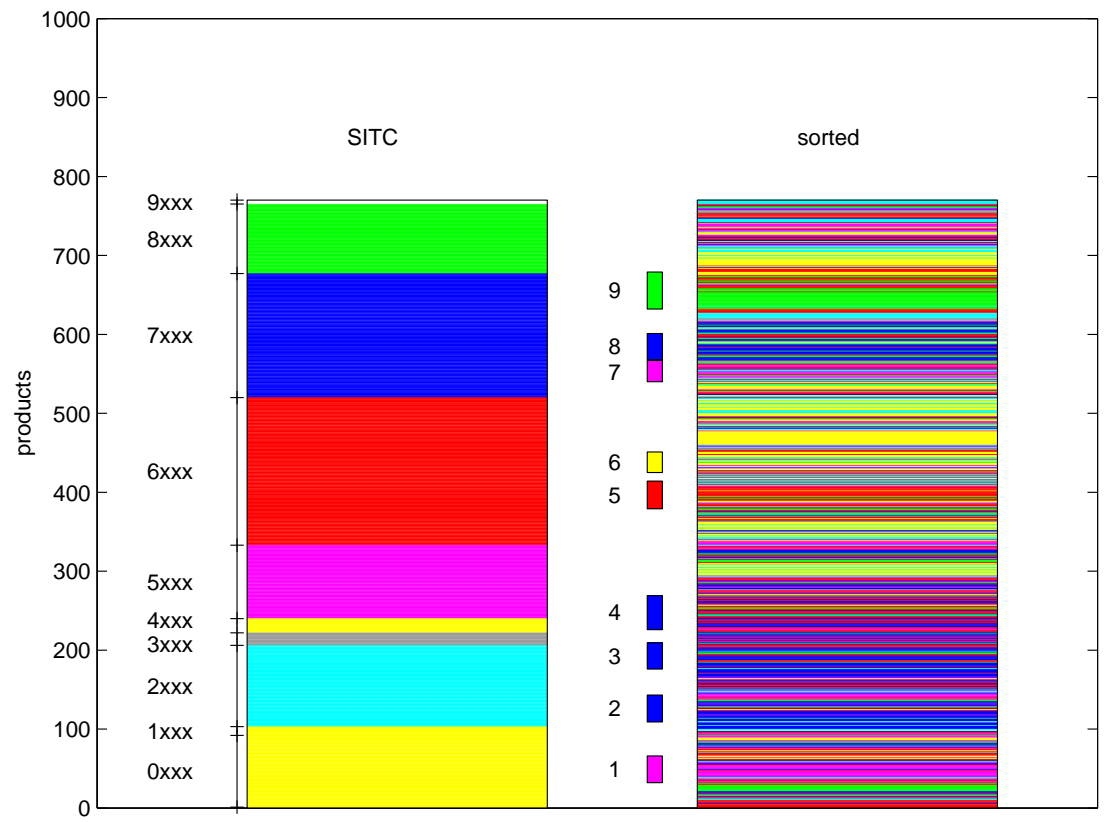

Fig. 4 Products ordered according to SITC codes (left) and ordered by the clustering algorithm (right). The vertical bars indicate the location of the 9 primary clusters. The colour code matches single-digit SITC.

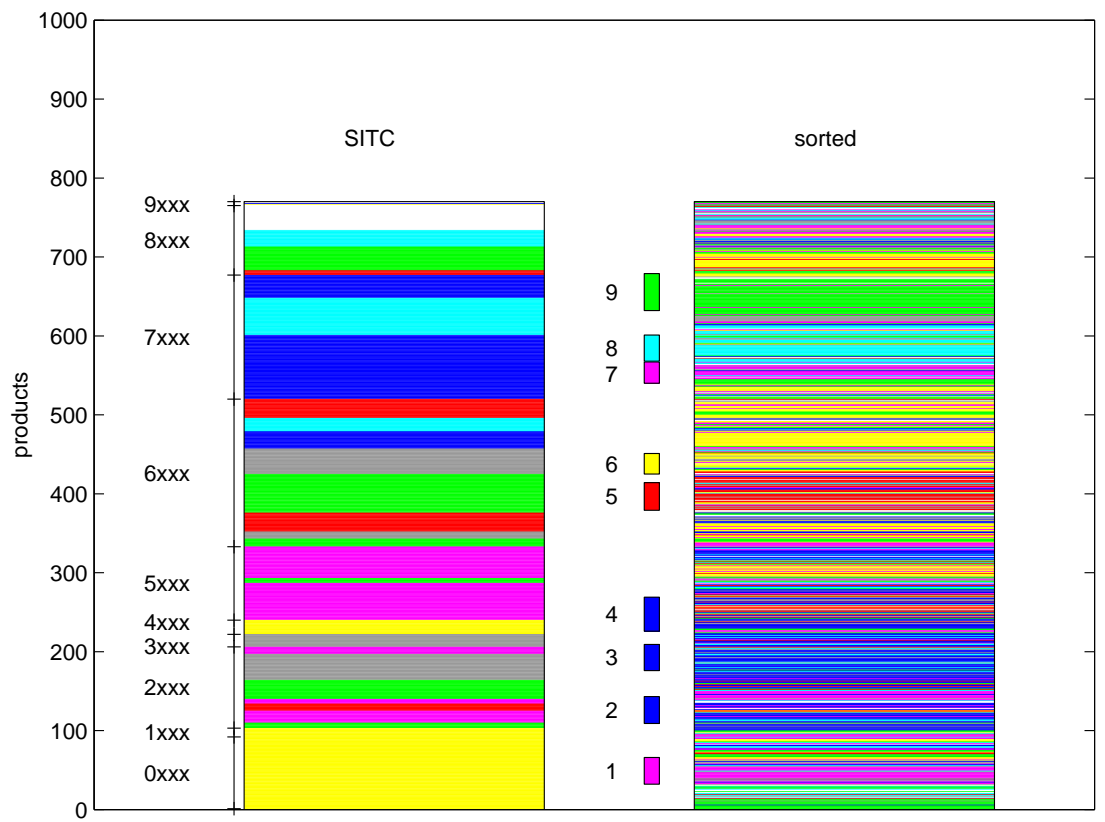

Fig. 5 The same as in the previous figure but with an alternative, refined colour coding. 


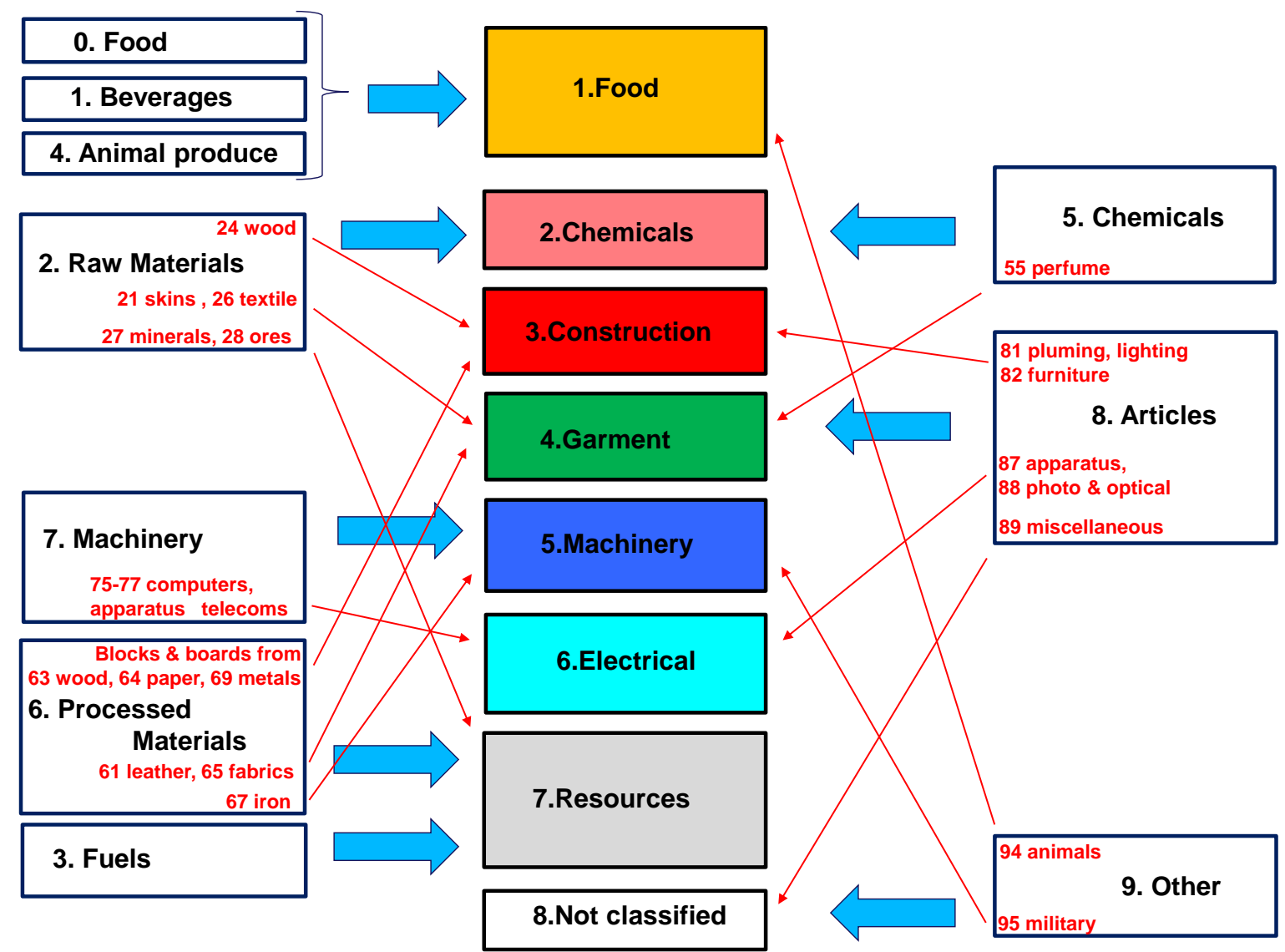

Fig. 6 Maping of SITC codes to cluster-related classification introducing the refined color coding, which is used in the previous figure. 

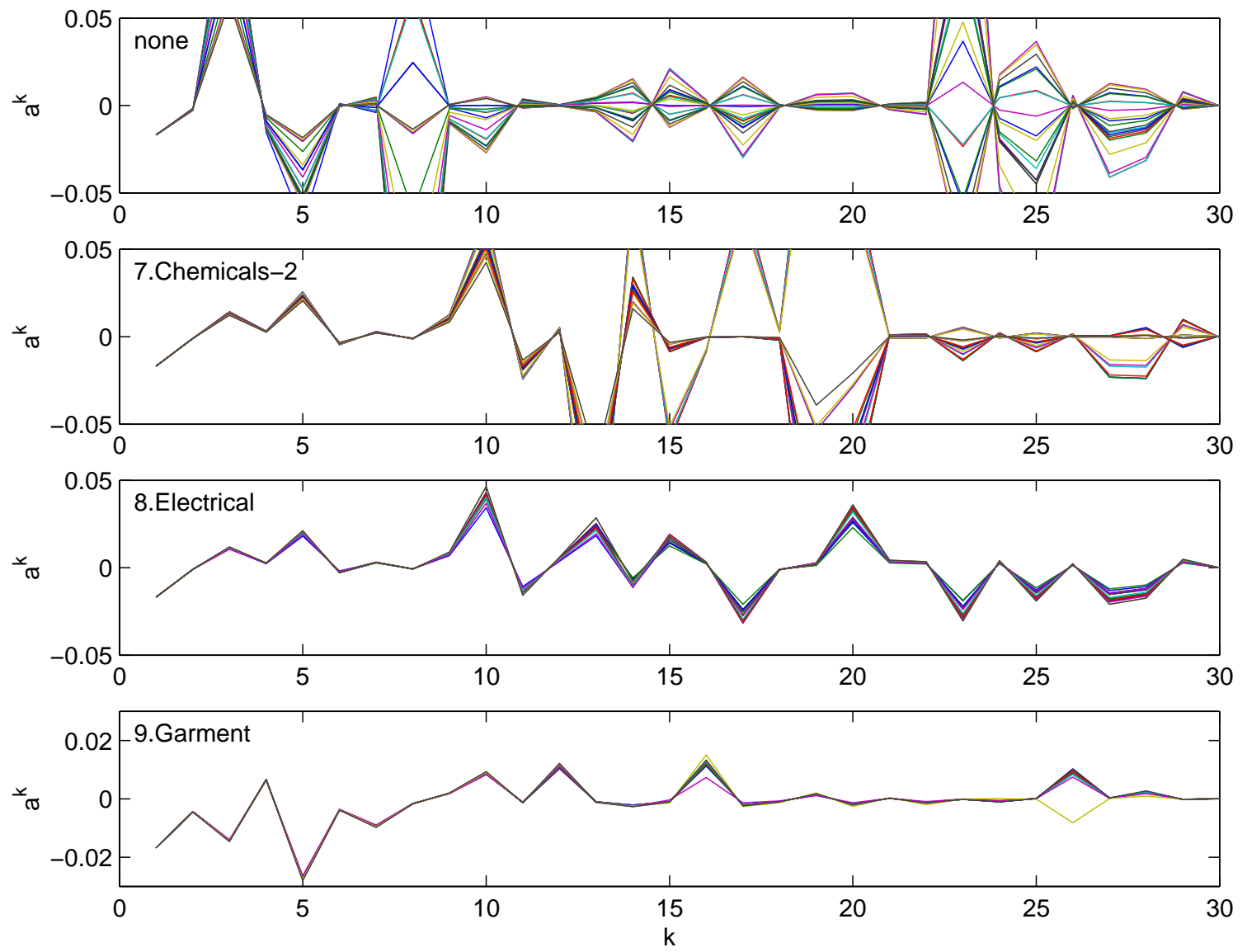

Fig. 7 Spectral representation for selected clusters. Each subfigure shows twenty lines corresponding to twenty nodes $i$ selected from a particular cluster plotted versus $k=1,2, \ldots, 30$. The top subfigure is shown for nodes $i=11,12, \ldots, 30$ (numbered after sorting), which do not correspond to any identified cluster. This subfigure is given for comparison. 


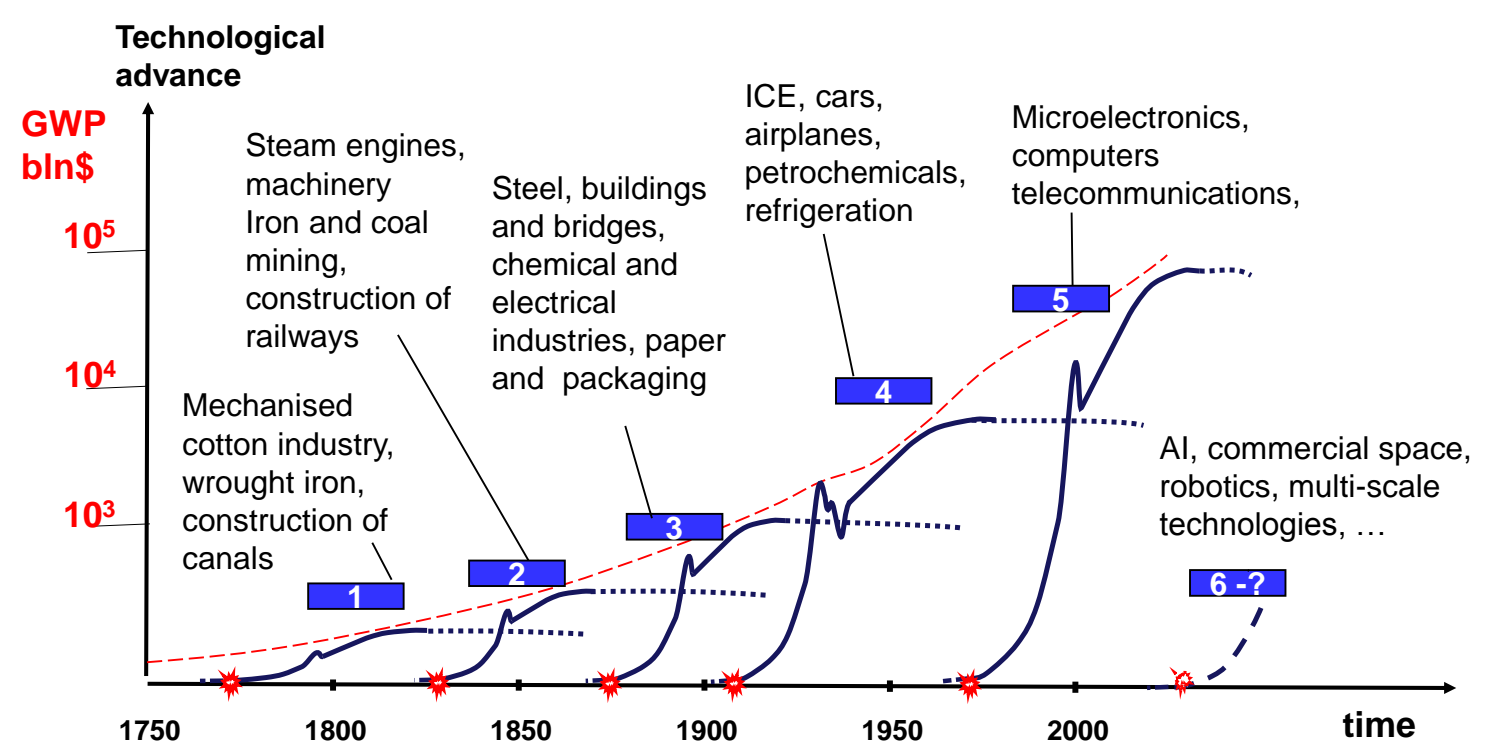

Fig. 8 Schematic of five technological surges due to Perez 2006. The figure also shows possible sixth surge and the estimate of Gross World Product using logarithmic scale. 